\title{
BIOSYNTHESIS OF SILVER
}

\section{NANOPARTICLES USING DRIED Agaricus bisporous EDIBLE MUSHROOM AND ITS ANTIMICROBIAL ACTIVITY AGAINST HUMAN PATHOGENS}

\author{
Sneha thomas \\ Department of Microbiology \\ Government science college \\ Nrupathunga road, Bangalore, India
}

\author{
Dr. Anisa athar \\ Department of Microbiology \\ Government science college \\ Nrupathunga road, Bangalore, India
}

\begin{abstract}
The aim of the present study was to synthesize silver nanoparticles (AgNPs) by using edible dried mushroom extract, $\underline{\text { Agaricus }}$ bisporus which is commonly called as the button mushroom. Mushrooms are used as nutritional and also for therapeutic purposes. In this study of biosynthetic method for producing silver nanoparticles have distinct advantages over chemical methods such as high biosafety and being ecofriendly and nontoxic to the environment. These functionalized silver nanoparticles also showed a noticeable antimicrobial activity against different clinically important pathogenic microorganisms. UV-vis spectroscopy result showed maximum adsorption at $200 \mathrm{~nm}$. The SEM analysis showed the morphology of the AgNPs as oval and spherical and the size of the particles ranges from 500nm. From the XRD, it was observed that the nanoparticles are silver with an average size ranged between $30 \mathrm{~nm}$ to $50 \mathrm{~nm}$. Hence, such type of synthesis methods for the production of nanostructured materials at lower cost and with natural energy may encourage production of functionalized AgNPs on industrial scale.
\end{abstract}

KEYWORDS; Silver nanoparticles, Agaricus bisporous, UV spectra, SEM, XRD, Antibacterial activity

\section{INTRODUCTION}

Nanoparticles are between 1 and 100 nanometers $(\mathrm{nm})$ in size with a surrounding interfacial layer. And the study of manipulating matter on an atomic scale is called Nanotechnology. This field provides a platform to modify and develop the important properties of metal in the form of nanoparticles having applications in diagnostics, biomarkers, cell labeling, contrast agents for biological imaging, antimicrobial agents, drug delivery systems and nano-drugs for treatment of various diseases. AgNPs are gaining more importance in the medical field as antimicrobials, sterilizers and testing tools for diagnosing and detecting sensitive biomolecules. When AgNPs enter inside a pathogen, the particle releases silver ions, thereby killing it. Hence, nano silver acts as an effective killing agent against all types of bacteria. AgNPs can be synthesized using various methods including chemical method. Although these methods can successfully produce silver nanoparticles in an efficient manner but it usually involves the use of toxic and hazardous chemicals which have several harmful effects on the environment and human health. Again, the final product requires more purification steps, as some of the by-products left behind during the process get adsorbed on the surface of AgNPs and can cause adverse effects during a medical application or treatment. These methods use expensive chemicals and usually require stabilizers to prevent agglomeration of AgNPs. Green synthesis does not involve the use of any toxic chemical, hence it is cost-effective, environmental friendly, zero energy based, doesn't contain any stabilizers and less time-consuming process. AgNPs synthesized by green process are highly compatible for pharmaceutical and other biomedical applications. The key phytochemicals responsible for converting silver ions into silver nanoparticles are found to be terpenoids, glycosides, alkaloids, etc., as identified in IR spectroscopic studies. Based on the above findings, the present study was carried out to examine the use of dried Agaricus bisporus in the form of extracts to prepare AgNPs and to prove its efficiency as an effective antimicrobial agent. 


\section{MATERIALS AND METHODS}

\section{Preparation of Crude extract of dried Agaricus bisporous mushroom}

Fresh mushrooms Agaricus bisporous (white button mushroom) were bought from commercial sources. $10 \mathrm{gm}$ of mushroom was weighed and washed thoroughly with distilled water and dried. It is then crushed and transferred to a beaker containing $100 \mathrm{ml}$ of distilled water. The mixture is stirred for $2 \mathrm{hrs}$ and then filtered using Whatman no. 1 filter paper. The resultant filtrate in the extract of mushroom used for the reduction of $\mathrm{Ag}+$ to $\mathrm{Ag} 0$. The extract of mushroom can be preserved for further experiments by storing in refrigerator.

\section{Standard preparation of Silver Nanoparticles}

In this synthesis of silver nanoparticle equal volume (1:1 ratio) of mushroom extract is mixed to $0.1 \mathrm{M} \mathrm{AgNO}$. The mixture is then incubated overnight in shaker at $150 \mathrm{rpm}$ at $37^{\circ} \mathrm{c}$.

The reduction of $\mathrm{AgNO} 3$ to $\mathrm{Ag}++$ nanoparticles is indicated by the color change from pale yellow to reddish brown.

\section{Characterization}

UV spectroscopy analysis of AgNP was shown between the ranges from 200 to $600 \mathrm{~nm}$.

SEM (scanning electron microscopy) and XRD (X -ray diffraction) techniques were used to know the morphology of the obtained AgNPs.

For these techniques the AgNPs sample were dried by flooding 100microlitre of the sample onto a $1 \mathrm{~cm}^{2}$ slide and keeping it in the hot air oven overnight. SEM was employed to measure the size of nanoparticles and XRD was to analyze the crystalline sized of the synthetized nanoparticles.

\section{Antibacterial activity of synthesized AgNP by Disc diffusion method:}

The antibacterial activities of the silver nanoparticles were determined by agar diffusion method. This method is used to find the antibacterial potential of silver nanoparticles against human pathogenic bacteria like Klebsiella, Staphylococcus, E. coli, Bacillus and Pseudomonas. The nutrient agar plates were prepared and the agar surface is inoculated with these test bacteria. Sterile discs are impregnated with $5 \mu \mathrm{l}$ solution of silver nanoparticle and were placed on the surface of the agar. The plate was incubated at $37^{\circ} \mathrm{C}$ for $24 \mathrm{hrs}$. After incubation, the plates were analyzed for the zones of inhibition and it was measured.

\section{RESULTS}

\section{Green synthesis of AgNP using dried Agaricus bisporus extract:}

Synthesis of AgNPs using the dried Agaricus bisporus extract was observed. When the extract was mixed with standard $0.1 \mathrm{M} \mathrm{AgNO}_{3}$ solution in 1:1 ratio and kept in shaker overnight there occurred to be a gradual change in the color from pale yellow to reddish brown in the solution. This change in color indicates the formation of AgNPs in the solution.

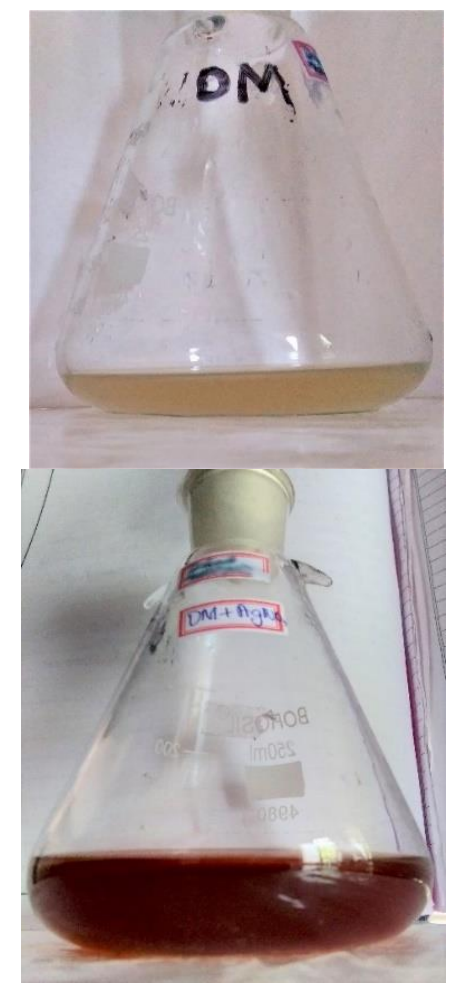

Before reduction of $\mathrm{Ag}^{2+}$ (pale yellow) after reduction of $\mathbf{A g}^{2+}$ (reddish brown)

\section{UV spectroscopy analysis:}

The UV spectroscopy analysis of the AgNP sample showed the maximum absorption at $200 \mathrm{~nm}$. The absorbance pattern obtained is shown in the Figure.

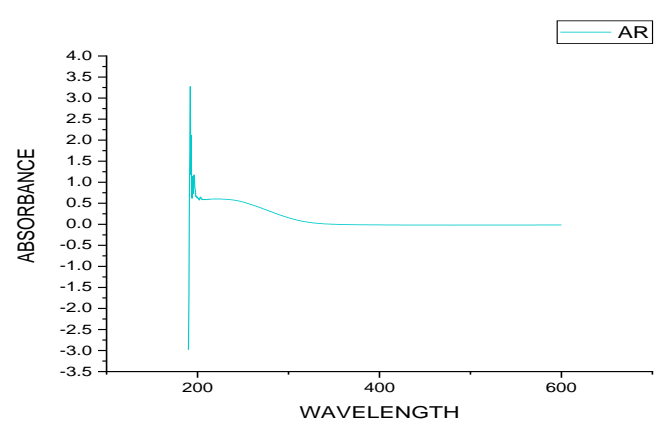


Figure of UV spectroscopy pattern

\section{SEM analysis:}

The SEM (scanning electron microscope) technique was used to analyze the particle shape and size of the synthesized silver nanoparticles. From this analysis, the shape of the nanoparticles obtained was found to be oval and spherical and the size ranges from $500 \mathrm{~nm}$.

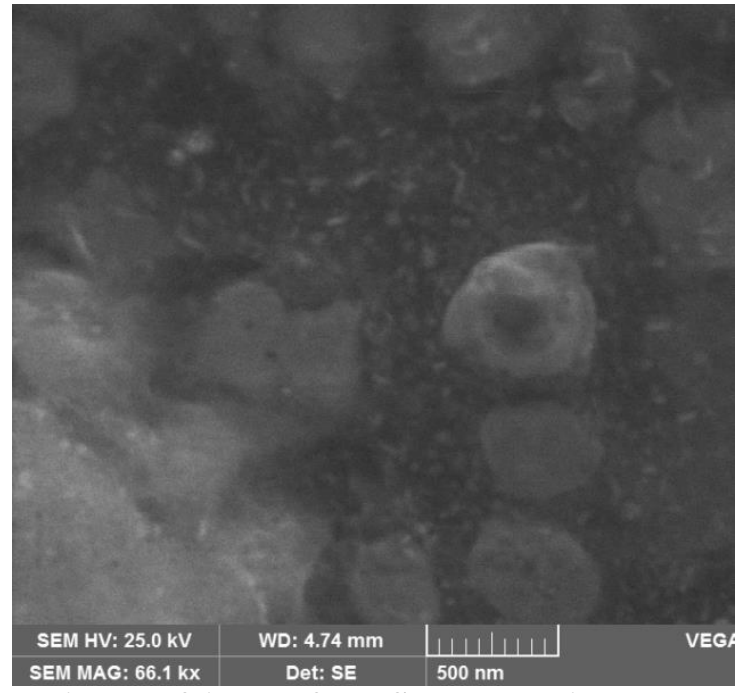

Picture of AgNPs from SEM analysis

\section{XRD analysis:}

From the XRD ( $\mathrm{X}$ - ray diffraction) the pattern of the obtained AgNPs is shown in Figure. The broadness of the peaks from the XRD pattern can be used to calculate the average crystalline size (D) of the AgNPs using the Scherrer's formula D $=\kappa \lambda / \beta \cos \theta$, where, $\kappa$ is constant (0.9) value known has shape factor, $\lambda$ is the wavelength of the $\mathrm{X}$ rays (1.5406), $\beta$ is FWHM (full width at half maximum) of the diffraction peaks and is the diffraction angle, $\theta$ is the Bragg angle $=2 \theta / 2$

The average crystalline size of the AgNPs calculated using Scherrer's formula is between the ranges $30 \mathrm{~nm}$ to $50 \mathrm{~nm}$

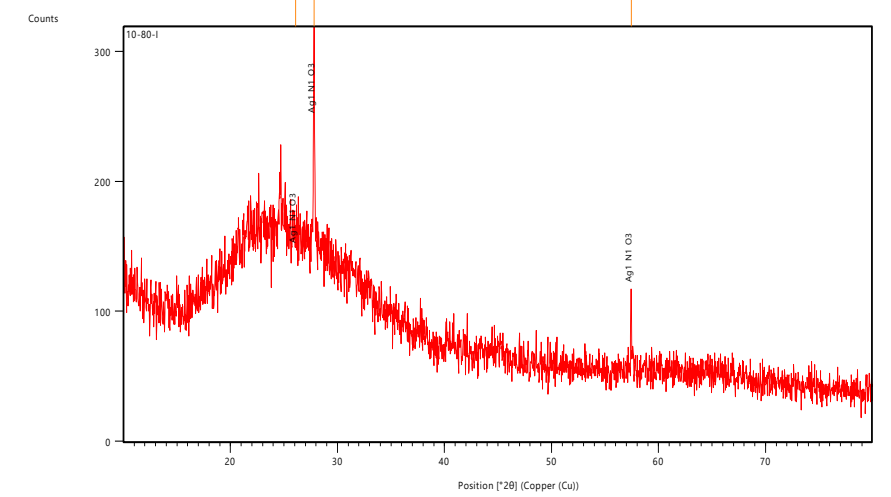

Figure of pattern obtained from XRD analysis

5. Antimicrobial activity of synthesized AgNPs:

The antibacterial potency of the obtained AgNPs was checked against Escherichia coli, Pseudomonas, Staphylococcus, Bacillus and Klebsiella. The zone of inhibition was observed and the diameter of the was tabulated as in the follows;

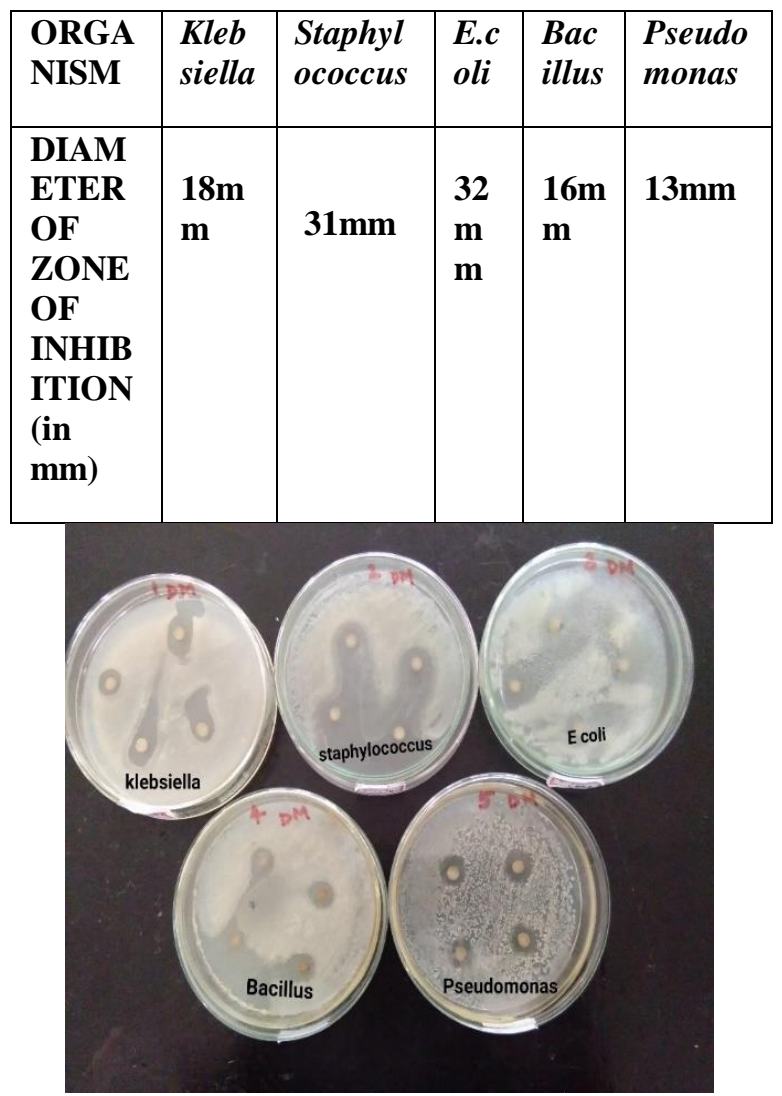

The plates showing zone of inhibition due to antibacterial activity of AgNPs 


\section{DISCUSSION}

Green synthesis of silver nanoparticles was followed to synthesize the silver nanoparticles. The silver nanoparticles were synthesized using the dried Agaricus bisporous extract.

The reduction of silver nitrate to silver nanoparticles was indicated by the color change from pale yellow to reddish brown. The color arises due to excitation of surface plasmon resonance (SPR) in the metal nanoparticles. The color change was observed within overnight of incubation. The surface plasmon resonance band occurs in the visible region of the light spectrum with absorbance peak was at $200 \mathrm{~nm}$.

Further the silver nanoparticles were characterized by SEM and XRD analysis. SEM study revealed a uniform arrangement of particles having size in the range of $500 \mathrm{~nm}$ and spherical and oval in shape. Whereas Thukkaram Sudhakar et.al.,2014[13] synthesized silver nanoparticles using Agaricus bisporous (non-dried mushroom) and obtained particle size in the range of $15-20 \mathrm{~nm}$ and of spherical shape. XRD pattern showed peak at $25^{\circ}, 28^{\circ}$ and $58^{\circ}$ indicating the crystalline nature of the reduced silver nanoparticles.

Anti-bacterial potency of the silver nanoparticles was tested against various bacteria. The synthesized Ag NPs shows an effective antibacterial activity against all the pathogens tested, almost correlating with the work of Thukkaram Sudhakar et.al.,2014 and Nithyaet.al., 2009 synthesized Ag NPs using Pleurotus sajorcaj

The antibacterial effect was studied on 5 different organisms by disc diffusion method. Klebsiella, Staphylococcus aureus, Escherichia coli, Bacillus, and Pseudomonas produced zone of inhibition of $18 \mathrm{~mm}, 31 \mathrm{~mm}, 32 \mathrm{~mm}, 16 \mathrm{~mm}$ and $14 \mathrm{~mm}$. In my studies silver nanoparticles showed a zone of $32 \mathrm{~mm}$ against Escherichia.coli, thus suggesting better anti-bacterial efficacy. The result suggests that silver nanoparticles interact with bacterial cell and affinity towards cysteine residues and thiols, thereby resulting in bactericidal effect and the bactericidal effect are based on the size of the nanoparticles synthesized. Thus, further studies can be carried out to reduce the particle size thereby increasing the bactericidal effect.

\section{CONCLUSION}

The present work is aimed at investigating the most efficient eco-friendly method for biosynthesis of silver nanoparticles using edible dried mushroom Agaricus bisporous and to do characterization of the synthesized silver nanoparticle for shape and size analysis followed by antibacterial study for determining the bio-toxicity of the Ag NPs.

Silver nanoparticles can be used effectively against multi-drug resistant bacteria due to their size and relatively large surface area in comparison to their volume makes easy to interact with substances and increases their antibacterial efficacy.

Silver may have an important advantage over conventional antibiotics in that kills all pathogenic microorganisms, and no organism has ever been reported to readily develop resistance to it. Several mechanisms have been proposed to explain the activity of silver ion or AgNPs on bacteria like: i) inactivation of respiratory chain, ii) disruption of cell membrane and leakage of its cellular contents, iii) binding to functional group of proteins causing protein denaturation and cell death, iv) blocking of DNA replication, and v) denaturation of enzymes which transport nutrients across bacterial cell membrane leading to death of microorganism. Studies have also demonstrated that silver ions interact with sulfhydryl (-SH) groups of proteins as well as the bases of DNA leading either to the inhibition of respiratory processes.

Application of incorporation of silver nanoparticles into or on the surface of products like cleaning sprays, skin creams, ATM buttons, and sports clothing etc. makes it attain antibacterial properties. The most important application of silver and silver nanoparticles is in topical ointments to prevent infection against burn and open wounds.

ACKNOWLEDGEMENT- The success and final outcome of this project required lot of guidance and assistance from many people and I am extremely privileged to have got this all along the completion of my project. All that I have done is only due to such supervision and assistance and I would not forget to thank them. I respect and thank Dr. Kavitha. B, Assistant professor HOD of Department of Microbiology, for providing me an opportunity to do the project work in MSc II semester and giving all support and guidance which made me complete the project duly. I am extremely thankful to her for providing such a nice support and guidance, although she had busy schedule managing the corporate affairs. I owe my deep gratitude to our guide Dr. Anisa Athar assistant professor who took keen interest on our project work and guided us all along, till the completion of our project work by providing all the necessary information for developing a good system. I would like to extend my gratitude to Mr. Shivaraj Kumar Assistant professor Department of Chemistry, for their suggestions and guidance. I am thankful to and fortunate enough to get constant 
support and guidance from all teaching staff of Department of Microbiology, Government Science College which helped us in successfully completing our project work.

\section{REFERENCES}

1. Santhosh Kumar T, Abdul Rahuman A, Rajakumar G, Marimuthu S, Bagavan A, Jayaseelan S, Adduz Zahir A, Elango G \& Kamaraj C, Parasitol Res, 2011, 108:693702 2.synthesis of silver nanoparticles using Nelumbonucifera leaf extract and its larvicidal activity against malaria and filariasis vectors.

2. Probin Phanjom, Elizabeth Zoremi D, Jahirul Mazumder, Moumita Saha, Sukanya Buzar Baruah, 2012, 3(2):73-79. Green synthesis of silver nanoparticles using leaf extract of Myricaesculenta. Int. J. of Nano Sci.and Nanotechnol.

3. Nagaraj B, Divya TK, Malakar Barasa, Krishnamurthy NB, Dinesh R, Negrila CC, 2012, 7(3):899-905. Phytosynthesis of gold nanoparticles using Caesalpiniapulcherrima (Peacock flower) flower extract and evaluation of their antimicrobial activities. Dig. J. of Nanomat. and Biostru.

4. A.T. Le, P.T. Huy, L.T. Tam, P. D. Tam, N. Hieu, T.Huy, 8(3) (2011) 278-290.

Novel silver nanoparticles: synthesis, properties and applications. Int. J. of Nanotechnol.

5. R. Singh, N.H. Singh, Medical Application of nanoparticles in Biological Imaging, Cell Labelling, 7(4) (2011) 489503. Antimicrobial Agents and Anticancer Nanodrugs. J. Biomed. Nanotechnol.

6. Fauss, E. 2008. The Silver Nanotechnology Commercial Inventory. University of Virginia.

7. L. Muthukrishnan, A. Nanda 2013. Genotoxic study of silver bio-nanoparticles toward Gram-positive and Gram-negative clinical isolates. J Pharm Res 6 (7), 725729. Thukkaram Sudhakar et al /Int.J. PharmTech Res.2014,6(5),pp 1718-1723. 1723.

8. J.A. Kiehlbauch, G.E. Hannet, M. Salfinger, W. Archinal, C. Monserrat, C. Carlyn, 38 (2000) 3341-48.Use of National Committee for Clinical laboratory Standards guidelines for disk diffusion susceptibility testing in New York state laboratories. J.Clin. Microbial.

9. Narasimha G, Praveen, Mallikarjuna K, Deva Prasad Raju, 2011. Vol. 2(1), p. 2936. Mushrooms (Agaricusbisporus) mediated biosynthesis of sliver nanoparticles, characterization and theirantimicrobial activity. Int.J.Nano Dim.

10. Ravishankar Bhat, Do Sung Huh, Raghunandan Deshpande, and Venkataraman, Photo-Irradiated Biosynthesis of Silver Nanoparticles Using Edible Mushroom Pleurotusflorida and Their Antibacterial Activity Studies. Hindawi Publishing Corporation, Bioinorganic Chemistry and Applications Volume 2011, 7 pages.

11. Anuradha Prakash, Seema Sharma, Naheed Ahmad, Ashok Ghosh, Bacteriamediated extracellular synthesis ;/of metallic nanoparticles. December, 2010, Vol. 1(5) p.071-079. International Research Journal of Biotechnology.

12. Anima Nanda, M Saravanan, 01/2013; 5:452-456. Biosynthesis of silver nanoparticles from Staphylococcus aureus and its antimicrobial activity against MRSA and MRSE. Nanomedicine Nanotechnology Biology and Medicine. 